\title{
Efecto del lavado con etilendiamina sobre las propiedades de las nanopartículas de óxido de estaño
}

\author{
A. MONTENEGRO HERNÁNDEZ ${ }^{1,}$, M. S. CASTRO ${ }^{2}$, J. E. RODRÍGUEZ PÁEZ ${ }^{1}$ \\ 1. Grupo de Ciencia y Tecnología de Materiales Cerámicos (CYTEMAC) Universidad del Cauca, \\ Calle 5 № 4-70 Popayán, Cauca, Colombia. \\ 2. Instituto de Investigaciones en Ciencia y Tecnología de Materiales (INTEMA, CONICET-UNMdP) \\ J.B. Justo 4302, B7608FDQ-Mar del Plata, Argentina.
}

\begin{abstract}
El óxido de estaño es un compuesto ampliamente utilizado en aplicaciones tecnológicas destacándose como catalizador, sensor de gas, en fabricación de varistores, dispositivos electrónicos, electrodos electrocatalíticos y celdas fotovoltaicas, entre otros. En el presente trabajo se utilizó el método de precipitación controlada (MPC) para sintetizar óxido de estaño de tamaño nanométrico, empleando como precursor sulfato de estaño. El control de las diferentes etapas del proceso de síntesis se realizó a través de los registros de variación de $\mathrm{pH}$, valoración potenciométrica, del sistema. Con base a esta información y el estudio de espectroscopía infrarroja realizada en la fase sólida, a diferentes valores de $\mathrm{pH}$, se propone un mecanismo de formación de las partículas donde las reacciones de hidrólisis y condensación son muy importantes. Se analizó, además, el efecto del lavado del precipitado con etilendiamina y se verificó la eliminación del anión sulfato con este proceso, obteniéndose al final óxido de estaño, $\mathrm{SnO}$ y $\mathrm{SnO}_{2}$, como principales fases cristalinas, a temperatura ambiente. Las características del óxido de estaño nanométrico se determinaron utilizando espectroscopía infrarroja de transmisión FTIR, difracción de rayos X (DRX), y microscopía electrónica de transmisión (MET).
\end{abstract}

Palabras clave: Síntesis, polvos cerámicos, precipitación controlada, nanopartículas, óxido de estaño, etilendiamina.

\section{Effect of washed with etilendiamine about the properties of the tin oxide nanoparticles}

Tin oxide, is a compound used in many technological applications among them as gas sensor and in the production of varistors, electronic devices, electrocatalitic electrodes and fotovoltaic cells, among others. In this work the controlled precipitation method was used to synthesize tin oxide of nanometric size using tin sulfate precursor. The control of different stages of the synthesis process was carried out through the variation of the system $\mathrm{pH}$ registrations, potentiometric titration,; This curve contain information on the different processes that take place in the system and it can be used to guarantee the process reproducibility. Additionally we propose a mechanism to explain the nucleation of solid phase in the solution. The hydrolysis and policondensation reactions are very important for this mechanism. Precipitate washing with an ethylendiamine solution permitted eliminated of $\mathrm{SO}_{4}^{2-}$ and it permitted to obtain the tin oxide as the principal crystalline phase at room temperature. The characteristics of the obtained ceramic powder were determined using infrared espectroscopy (FTIR), X-ray diffraction (XRD), and transmission electron microscopy (TEM).

Keywords: Tin oxide; synthesis; controlled precipitation; nanoparticles; ethylendiamine..

\section{INTRODUCCIÓN}

La síntesis de nano-partículas es un campo de gran interés científico y tecnológico, debido a las propiedades físicas y químicas que ellas presentan $(1,2)$. La estructura cristalina, el tamaño y forma de las partículas y las características superficiales dependen de la ruta de síntesis utilizada para su obtención. Esto exige conocer más en detalle los métodos de síntesis de nano-partículas para asegurar la calidad del polvo cerámico, sus propiedades y por lo tanto las características del dispositivo final que con el se fabrique. La descripción del método de síntesis debería brindar información sobre los fenómenos fisicoquímicos que ocurren durante la formación de las nano-partículas, pero lo que realmente se requiere es conocer el mecanismo de formación de las partículas ya que con este conocimiento es posible tener un mayor control sobre las propiedades del polvo cerámico final $(3,4)$.

El óxido de estaño es un material semiconductor transparente con gran estabilidad química y mecánica. Este óxido tiene una fase estable, conocida como casiterita, y otra denominada romarchita, $\mathrm{SnO}$ (5). Los principales yacimientos de este mineral se encuentran en Brasil, Perú y Bolivia (6).Al igual que el óxido de cinc, el $\mathrm{SnO}_{2}$ es un semiconductor con banda prohibida ancha y presenta una adecuada combinación de propiedades químicas, electrónicas y ópticas que lo hacen útil como material para sensores de gas (7), varistores (8), catalizadores (9), dispositivos optoelectrónicos, electrodos electrocatalíticos y celdas fotovoltaicas (10-12).

Diferentes métodos de síntesis han sido utilizados para la obtención del $\mathrm{SnO}_{2}$. Zhang y Gao (13) sintetizaron 
nanopartículas de $\mathrm{SnO}_{2}$ utilizando un método químico novedoso partiendo de estaño granulado. Para lograrlo adicionaron, cuidadosamente, una solución $8 \mathrm{~mol} \mathrm{~L}^{-1}$ de $\mathrm{HNO}_{3}$ a una mezcla de $3 \mathrm{~g}$ de estaño granulado con $10 \mathrm{~g}$ de ácido cítrico, hasta que obtuvieron una solución clara. Posteriormente adicionaron $\mathrm{NH}_{4} \mathrm{OH}$, gota a gota, hasta que el sistema alcanzó un valor de $\mathrm{pH}$ 8. Después de un proceso de reflujo, a $100^{\circ} \mathrm{C}$ durante $2 \mathrm{~h}$, obtuvieron partículas de $\mathrm{SnO}_{2}$ en el rango de 2.8 y $5.1 \mathrm{~nm}$ con una superficie específica entre 289 y $143 \mathrm{~m}^{2} \mathrm{~g}^{-1}$.

Otro método de síntesis empleado ampliamente es el de sol-gel. Con este proceso se pueden obtener polvos cerámicos a través del crecimiento de oxo-polímeros del metal en un solvente $(14,15)$. S. de Monredon et al. (16) utilizaron isopropóxido de estaño disuelto en etanol absoluto, bajo una atmósfera seca, y utilizando como acomplejante acetilacetona, AcacH, el cual fue adicionado al sistema bajo continua agitación. La hidrólisis de la solución fue realizada usando soluciones acuosas ácidas (ácido p-toluenosulfonico) que se dejaron envejecer, durante $24 \mathrm{~h}$ en botellas de vidrio fuertemente sellados, en un horno a $60^{\circ} \mathrm{C}$. A través de este método de síntesis se obtuvieron partículas de óxido de estaño monodispersadas con tamaño entre 1 y $2 \mathrm{~nm}$.

Utilizando el método de precipitación homogénea Song y Kang (17) sintetizaron $\mathrm{SnO}_{2}$ con un tamaño de partícula entre 3 y $4 \mathrm{~nm}$ de diámetro, una distribución de tamaño de partícula estrecho y un valor de superficie específica entre 24 y $44 \mathrm{~m}^{2} / \mathrm{g}$. Para ello disolvieron urea en una solución acuosa de $\mathrm{SnCl}_{4} .5 \mathrm{H}_{2} \mathrm{O}(0.01 \mathrm{M})$. La solución fue hidrolizada a $90^{\circ} \mathrm{C}$ durante 4 h y se enfrió utilizando agua con hielo. El precipitado se separó del sistema utilizando ultracentrifugación de la solución, el cual se secó a $100^{\circ} \mathrm{C}$ durante $24 \mathrm{~h}$. El polvo seco se trató térmicamente a diferentes temperaturas siendo la más favorable, para las características requeridas del polvo cerámico, la de $600^{\circ} \mathrm{C}$.

Como se puede deducir de lo enunciado, el método de síntesis del óxido de estaño tiene un gran efecto sobre el tamaño de partícula, la morfología de la misma y sus propiedades semiconductoras; hay en la literatura $(18,19)$ estudios que tratan explícitamente esta relación. Aunque en los trabajos sobre la obtención de $\mathrm{SnO}_{2}$ se realiza una descripción adecuada de los métodos de síntesis, en ellos no se hace mención a los mecanismos fisicoquímicos que llevan a la formación de las partículas. Además en ellos no se considera la formación de romarchita, $\mathrm{SnO}$, polvo negro insoluble en agua, pero que si lo es en ácidos y bases fuertes, donde la celda unitaria es tetragonal con una geometría de coordinación piramidal cuadrada (20), los parámetros de red son: $a=b=$ $3,8029 \AA$ y c $=4,8382 \AA$, tiene un nivel de energía en el rango de $2,5-3 \mathrm{eV}$, presenta un calor de formación $\Delta \mathrm{H}=-138 \mathrm{cal} / \mathrm{mol}$ a $298^{\circ} \mathrm{K}$ (21) y tiene una entalpía de formación de -285,9. (20)., Este compuesto debería estudiarse con mayor cuidado dadas sus posibles aplicaciones tecnológicas tales como: agente reductor e intermediario químico (22), componente del vidrio (23), celdas de litio (24), entre otras.

En este trabajo se estudió la obtención de óxido de estaño a partir de soluciones acuosas de sulfato de estaño, utilizando como precipitante hidróxido de amonio $\left(\mathrm{NH}_{4} \mathrm{OH}\right)$. Con base en las curvas de valoración potenciométrica se determinaron las diferentes etapas del proceso y se analizaron los fenómenos fisicoquímicos que ocurren durante la adición del precipitante. Específicamente, se estudió el efecto de lavar varias veces el precipitado, utilizando una solución de $0.005 \mathrm{M}$ de etilendiamina, sobre la presencia de sulfatos en el sólido y las transformaciones de fases que ocurren durante este proceso hasta obtener óxido de estaño, una mezcla de $\mathrm{SnO}_{2}$ y SnO, a temperatura ambiente. Los sólidos obtenidos a diferentes valores de $\mathrm{pH}$ del sistema, y al final del proceso, se caracterizaron con espectroscopia infrarroja (FTIR), difracción de rayos X (DRX) y Microscopía Electrónica de transmisión (MET)..

\section{PROCEDIMIENTO EXPERIMENTAL}

\subsection{Síntesis de los polvos cerámicos de óxido de estaño.}

Se prepararon soluciones acuosas a diferente concentración de sulfato de estaño $\left(\mathrm{SnSO}_{4}\right.$-Fisher Scientific 95\%), con $0.1 \mathrm{M}$ de acido nítrico $\left(\mathrm{HNO}_{3}\right.$-Carlo Erba $\left.99 \%\right)$. Las soluciones se agitaron constantemente a 200 r.p.m., a temperatura ambiente, hasta que no se observaron partículas del precursor en suspensión ni sedimentadas. Luego se adicionó hidróxido de amonio ( $\mathrm{NH}_{4} \mathrm{OH}-$ Mallincrodt $28 \%$ ) a una velocidad de $0.034 \mathrm{mLs}^{-1}$ utilizando para ello un dosificador (Metrohm Dosimat 685). La variación del $\mathrm{pH}$ del sistema se registró con un pH-metro (Metrohm 744), con electrodo de vidrio. A medida que se adicionó el $\mathrm{NH}_{4} \mathrm{OH}$ al sistema, el valor del $\mathrm{pH}$ de la solución se grafico en función del volumen adicionado de precipitante, $\mathrm{NH}_{4} \mathrm{OH}$, obteniéndose así las curvas de valoración potenciométrica del sistema de estaño.

La suspensión obtenida se filtró al vacío para eliminar el solvente y arrastrar los iones sulfato disueltos en él; el sólido húmedo obtenido de la suspensión con valor de $\mathrm{pH}$ 6.4, se redispersó en una solución acuosa $0.005 \mathrm{M}$ de etilendiamina $\left(\mathrm{C}_{2} \mathrm{H}_{8} \mathrm{~N}_{2}\right.$-Merck-Schuchardt 99\%) utilizando un equipo de dispersión de alta cizalla, Ultraturrax IKA Mod. T-50, sometiendo la mezcla a una velocidad de agitación de 4000 r.p.m, durante 3 minutos. La suspensión obtenida se dejó reposar durante 24 horas, luego se filtró; el sólido húmedo que se obtuvo se volvió a redispersar en la solución con etilendiamina y se adiciono ácido nítrico para llevar nuevamente el sistema a un $\mathrm{pH}$ de 6.4. Este proceso, denominado lavado, se repitió 10 veces. Con el fin de determinar el efecto del lavado con etilendiamina, sobre las propiedades del material en polvo, se tomaron muestras sólidas en diferentes instantes del proceso; para distinguir adecuadamente las muestras que se estudiaron se utilizo una notación, donde se acompaña a la letra " $\mathrm{L}$ " con un número que indica la cantidad de lavados con etilendiamina.

El producto obtenido, al finalizar el lavad o correspondiente, se secó en una estufa a $60^{\circ} \mathrm{C}$ durante 24 horas, luego se maceró en un mortero de ágata y el polvo obtenido se caracterizó, utilizando las técnicas mencionadas.

\subsection{Caracterización de los polvos cerámicos obtenidos.}

Los polvos obtenidos fueron caracterizados con difracción de rayos $\mathrm{X}(\mathrm{DRX})$, para determinar las fases cristalinas presentes en los sólidos; el equipo empleado fue un difractómetro marca Rigaku 2200 utilizando la radiación $\mathrm{CuK}_{\alpha^{\prime}}$ en el rango de 20 a $70^{\circ}(2 \theta)$. La espectroscopía infrarroja con transformada de Fourier se empleó con el fin de conocer los grupos funcionales que existen en las diferentes fases sólidas que se forman durante el proceso de síntesis; para ello se utilizó el espectrofotómetro FTIR Nicolet Modelo IR200 spectrometer. Por último, para conocer el tamaño y morfología de las partículas se empleó el microscopio electrónico de transmisión 
Jeol JEM-1200 EX.

\section{RESULTADOS Y DISCUSIÓN}

\subsection{Valoración potenciométrica.}

La curva de valoración potenciométrica de la figura 1(a), obtenida para una disolución $0.3 \mathrm{M}$ de $\mathrm{SnSO}_{4}$ y 0.1 de $\mathrm{HNO}_{3}$ al adicionar el agente precipitante $\left(\mathrm{NH}_{4} \mathrm{OH}\right)$, presenta 4 regiones bien definidas si se considera la variación de la pendiente de la curva; mientras la región A presenta una variación apreciable en el valor de $\mathrm{pH}$, la $\mathrm{B}$ es prácticamente plana, indicando un alto consumo de $\mathrm{OH}^{-}$por parte del sistema. La región $\mathrm{C}$ muestra un cambio abrupto de la pendiente de la curva y en la región $\mathrm{D}$ el valor de la pendiente es muy pequeño y corresponde a la saturación de la disolución.

a)
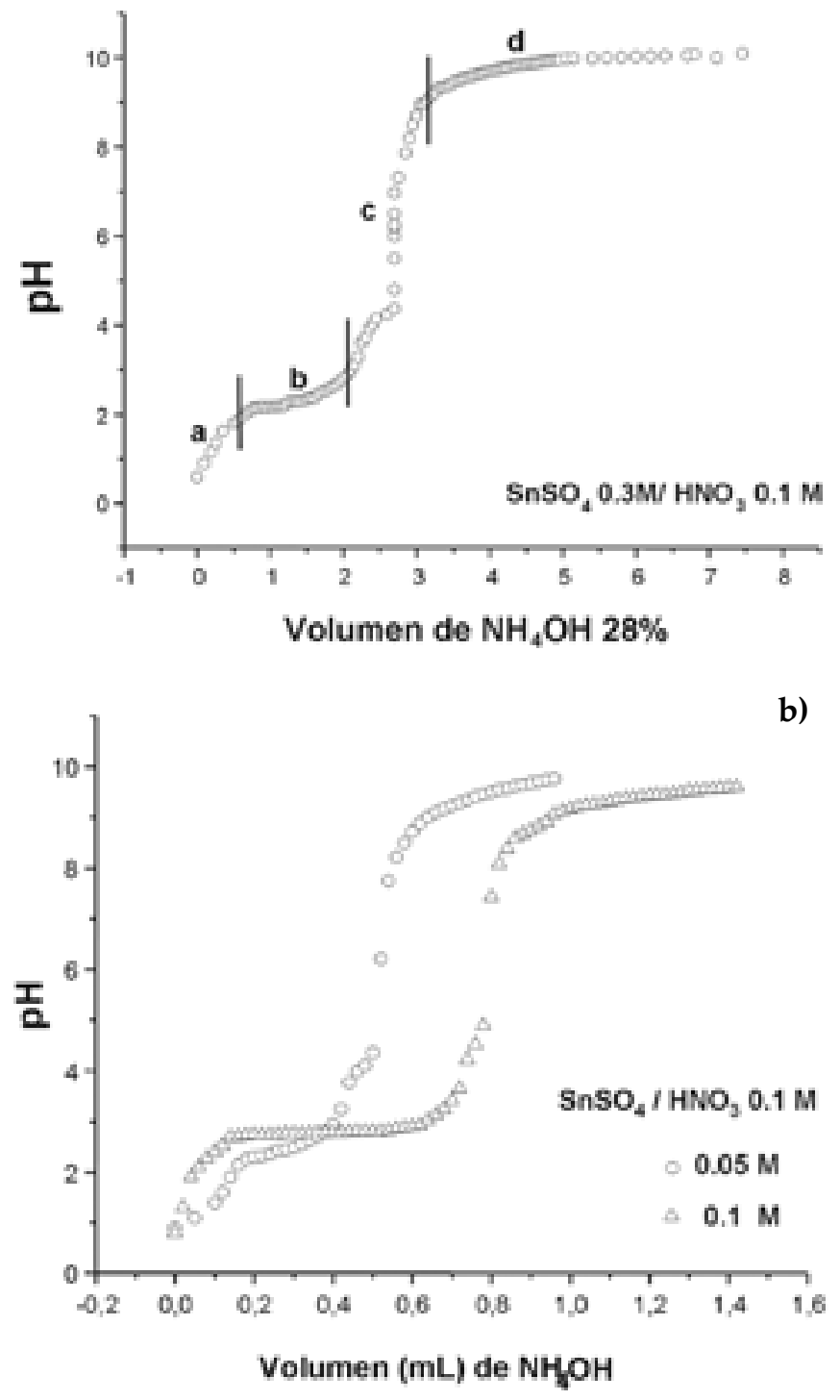

Fig. 1- Curvas de valoración potenciométrica correspondientes al sistema $\mathrm{Sn}\left(\mathrm{SO}_{4}\right)-\mathrm{HNO}_{3}-\mathrm{H}_{2} \mathrm{O}$ : (a) para una concentración 0.3M de $\mathrm{SnSO}_{4}$ y (b) diferentes concentraciones de sulfato de estaño.
$\mathrm{Al}$ disolver $\mathrm{SnSO}_{4}$ en agua se presenta una disociación parcial del precursor que se puede expresar a través de una reacción del siguiente tipo (25):

$$
\left[\mathrm{Sn}\left(\mathrm{SO}_{4}\right)_{\mathrm{N}}\right]^{-\mathrm{z}}+\mathrm{H}_{2} \mathrm{O} \rightarrow\left[\mathrm{Sn}\left(\mathrm{SO}_{4}\right)_{\mathrm{N}-\mathrm{h}}\left(\mathrm{OH}_{2}\right)_{\mathrm{h}}\right]^{-(\mathrm{z}-\mathrm{h})}+\mathrm{hSO}_{4}^{2-}
$$

donde la especie $\left[\mathrm{Sn}\left(\mathrm{SO}_{4}\right)_{\mathrm{N}}\right]^{-\mathrm{z}}$ representa el poliedro de coordinación del $\mathrm{Sn}^{2+}$ en solución. Si se produjera una disociación completa del precursor, en el sistema ocurriría la siguiente reacción:

$$
\mathrm{SnSO}_{4}+\mathrm{H}_{2} \mathrm{O} \rightarrow \mathrm{Sn}^{2+}(\mathrm{aq})+\mathrm{SO}^{2-}(\mathrm{aq})
$$

que generaría un complejo acuo de estaño el cual podría seguir interactuando con el agua a través de reacciones de hidrólisis (15):

$$
\left[\mathrm{Sn}\left(\mathrm{OH}_{2}\right)_{\mathrm{N}}\right]^{\mathrm{z+}}+\mathrm{H}_{2} \mathrm{O} \rightarrow\left[\mathrm{Sn}(\mathrm{OH})_{\mathrm{h}}\left(\mathrm{OH}_{2}\right)_{\mathrm{N}-\mathrm{h}}\right]^{(\mathrm{z}-\mathrm{h})}+\mathrm{H}_{3}{ }^{+} \mathrm{O}
$$

Adicional a la reacción 3, puede ocurrir la hidrólisis espontánea de la especie sulfatada, producto de la reacción 1, y que se puede expresar así (25):

$\left.\left[\mathrm{Sn}\left(\mathrm{SO}_{4}\right)_{\mathrm{N}-\mathrm{h}}\left(\mathrm{OH}_{2}\right)_{\mathrm{h}}\right]^{-(\mathrm{z}-2)}+\mathrm{H}_{2} \mathrm{O} \rightarrow \mathrm{Sn}\left(\mathrm{SO}_{4}\right)_{\mathrm{N}-\mathrm{h}}\left(\mathrm{OH}_{2}\right)_{\mathrm{h}-\mathrm{l}}(\mathrm{OH})\right]^{(\mathrm{z}-3)}+\mathrm{H}_{3}^{+} \mathrm{O}$

obteniéndose como un producto de la reacción protones, $\mathrm{H}_{3}{ }^{+} \mathrm{O}$, los cuales ocasionan la disminución del $\mathrm{pH}$ del sistema, tal como se observa experimentalmente al disolver el precursor de estaño en agua.

Por otro lado, si se observa la figura 1(a), en la región A de la curva ocurre inicialmente un leve incremento en el valor del $\mathrm{pH}$ que se debe, principalmente, a la neutralización del $\mathrm{HNO}_{3}$ por el $\mathrm{NH}_{4} \mathrm{OH}$ que se adiciona, produciéndose nitrato de amonio $\left(\mathrm{NH}_{4} \mathrm{NO}_{3}\right)$. Debido a que el ion sulfato se enlaza fuertemente al catión, se pueden conformar sulfatos básicos de estaño a través de la siguiente reacción:

$$
\mathrm{SnSO}_{4}+\mathrm{nNH}_{4} \mathrm{OH} \leftrightarrow \mathrm{Sn}(\mathrm{OH})_{\mathrm{n}}\left(\mathrm{SO}_{4}\right)_{1-\mathrm{n} / 2}+\mathrm{n} / 2\left(\mathrm{NH}_{4}\right)_{2} \mathrm{SO}_{4}
$$

En esta región, aproximadamente a $\mathrm{pH}$ 2, se forma una fase sólida transitoria que se disuelve al agitar el sistema, retomando la transparencia y homogeneidad inicial.

En la región $\mathrm{B}$ ocurre un alto consumo del $\mathrm{OH}^{-}$suministrado por el $\mathrm{NH}_{4} \mathrm{OH}$. En esta zona de la curva debe presentarse, principalmente, la conformación de especies poli-nucleares del tipo $\left[\mathrm{Sn}_{2}(\mathrm{OH})_{2}\right]^{2+},\left[\mathrm{Sn}_{3}(\mathrm{OH})_{4}\right]^{2+}$ y $\left[\mathrm{Sn}_{3}\left(\mathrm{SO}_{4}\right)(\mathrm{OH})_{4}\right]$, etc., como resultado del desarrollo de las reacciones de condensación que experimentan las especies hidrolizadas de estaño $(15,26,27)$. Los complejos tipo oxi-hidroxo que se pueden formar en esta región presentan una composición cercana a $3 \mathrm{SnO} . \mathrm{H}_{2} \mathrm{O}$, los cuales contienen agrupaciones de poliedros $\mathrm{Sn}_{6} \mathrm{O}_{8}$ que se unen entre sí por enlaces de hidrógeno dando como resultado una formula estructural $\mathrm{Sn}_{6} \mathrm{O}_{8} \mathrm{H}_{4}$, compuesto que puede formarse a partir de la siguiente reacción de condensación (26):

$$
2\left[\mathrm{Sn}_{3}(\mathrm{OH})_{4}\right]^{2+}+4 \mathrm{OH}^{-} \rightarrow\left[\mathrm{Sn}_{6} \mathrm{O}_{8} \mathrm{H}_{4}\right]+4 \mathrm{H}_{2} \mathrm{O}
$$

Las especies poli-nucleares que se conforman interactuaran 
para formar los embriones y luego los núcleos de la fase sólida. Es por esto que en esta región empiezan a formarse partículas que generan una suspensión de color blanco, que no se disuelven, alrededor de un valor de $\mathrm{pH} 3$; al seguir adicionando $\mathrm{NH}_{4} \mathrm{OH}$ al sistema éste se torna "lechoso", con partículas coloidales en suspensión. Como lo indicara el difractograma de rayos $\mathrm{X}$ de la muestra sólida obtenida a $\mathrm{pH}$ 3.9, en ella existe casiterita que se debe producir a través de la siguiente reacción (27):

$$
\mathrm{SnO}+\mathrm{H}_{2} \mathrm{O} \rightarrow \mathrm{SnO}_{2}+\mathrm{H}_{2}
$$

En la región $\mathrm{C}$ ocurre un incremento abrupto del $\mathrm{pH}$. En esta región la formación de los complejos y compuestos de estaño, y por lo tanto de los núcleos de fase sólida, debe disminuir notablemente. Alrededor de $\mathrm{pH}$ 5, el sistema se conviertió en una suspensión altamente viscosa y comienzó a cambiar de color, pasando de un color blanco a uno crema claro; estas características se mantienen hasta un $\mathrm{pH}$ de 7,5. Por encima de este valor la viscosidad del sistema disminuyo, pero mantuvo su turbidez, cambio de color pasando de crema claro a crema oscuro y posteriormente, con la adición del $\mathrm{NH}_{4} \mathrm{OH}$, a un color marrón claro; este color lo mantuvo por la suspensión en la región D. En esta última región, la formación de aglomerados debe ser el fenómeno más importante.

La figura 1(b) muestra como se modifican las curvas de valoración potenciométrica al variar la concentración del precursor de estaño, $\mathrm{SnSO}_{4^{\prime}}$ en el sistema. En todas ellas son evidentes las cuatro regiones de la figura 1(a), descritas anteriormente. La extensión de las regiones A y B se incrementa a medida que aumenta la concentración del precursor, esto debido a que la formación de núcleos de fase sólida debe ser mayor al incrementarse la concentración de $\mathrm{SnSO}_{4}$ en el sistema.

\subsection{Caracterización de la fase sólida de las suspensiones.}

Para determinar el efecto del $\mathrm{pH}$ y del proceso de lavado con una solución $0.005 \mathrm{M}$ de dietilamina, sobre la naturaleza de los sólidos que se forman en el seno de la disolución, se analizaron con FTIR y DRX, muestras sólidas obtenidas al filtrar suspensiones en diferentes instantes del proceso de síntesis de la materia prima.

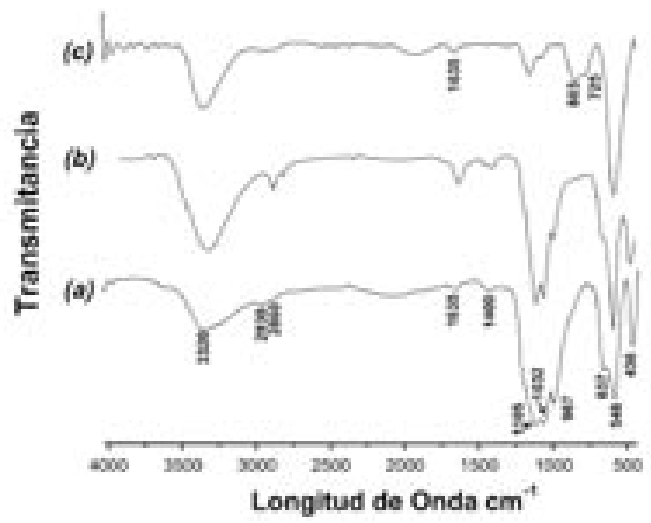

Fig. 2- Espectros FTIR de muestras sólidas obtenidas a diferentes valores de pH: (a) 3.97, (b) 6.40 y (c) 9.35 para el sistema de sulfato de estaño $0,3 \mathrm{M}$.

\subsubsection{Espectroscopía Infrarroja con transformada de Fourier} (FTIR).

Los espectros FTIR correspondientes a muestras sólidas obtenidas de la suspensión de estaño a diferentes valores de $\mathrm{pH}$, para una concentración $0,3 \mathrm{M}$ de $\mathrm{SnSO}_{4^{\prime}}$, se indican en la figura 2.

En los espectros de la figura 2, son evidentes las bandas del agua, 3320 y $1635 \mathrm{~cm}^{-1}$, del grupo funcional C-H, 2930 y $2860 \mathrm{~cm}^{-1}(28,29)$, y del N-H a $1400 \mathrm{~cm}^{-1}$. Las bandas que se encuentran entre 1200 y $900 \mathrm{~cm}^{-1}$ dan información sobre el enlace que forma el estaño y el sulfato presente en el sistema, además son las que presentan variaciones más evidentes. A bajos valores de $\mathrm{pH}$ se presentan enlaces de naturaleza monodentada del sulfato que se asocian a las bandas a 1105 , 1032 y $967 \mathrm{~cm}^{-1}(30,31)$. Además, las bandas a 436 y $546 \mathrm{~cm}^{-1}$ corresponden a los enlaces $\mathrm{Sn}-\mathrm{OH}$ y $\mathrm{Sn}-\mathrm{O}$ y las bandas a 637, 725 y $803 \mathrm{~cm}^{-1}$ están asociadas al enlace Sn-O-Sn $(32,33)$.

Para determinar el efecto que tiene en el sistema 0,3 M de $\mathrm{SnSO}_{4}$ los procesos de lavado, con una solución $0.005 \mathrm{M}$ de etilendiamina, se tomaron espectros FTIR a muestras sólidas obtenidas al filtrar la suspensión después de los diferentes lavados, estos se indican en la figura 3.

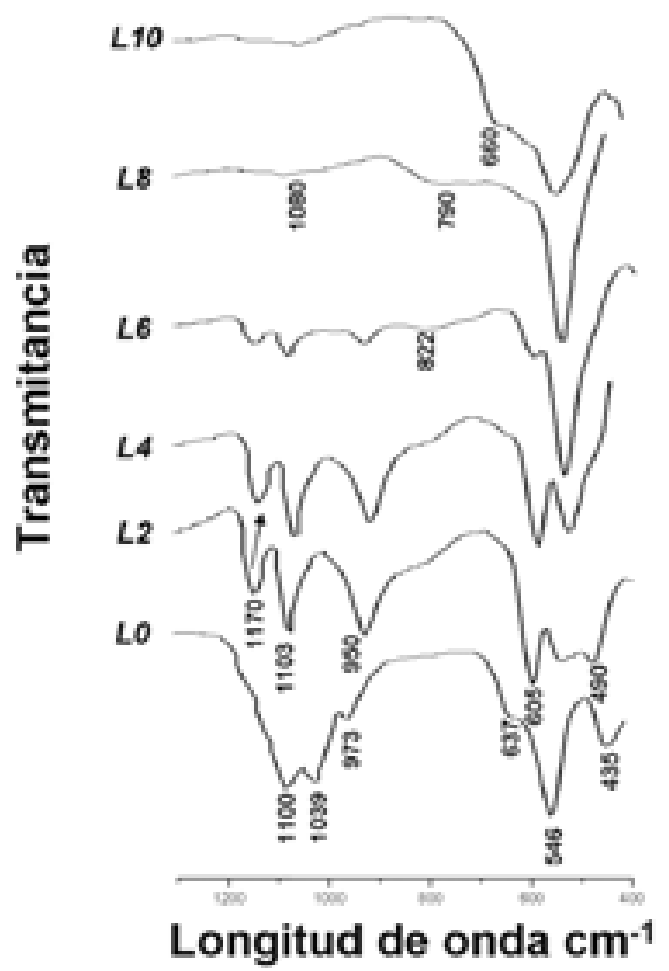

Fig. 3- Espectros FTIR correspondientes a muestras sólidas obtenidas del sistema $0.3 \mathrm{M}$ de sulfato de estaño a un valor de $\mathrm{pH}$ de 6.40 donde se indican el efecto de los lavados con etilendiamina.

Como se observa en la figura 3, para el segundo y cuarto lavado es evidente la presencia del sulfato en el sistema, pero a medida que se avanza en el proceso de lavado, sexto lavado, las bandas asociadas a este se debilitan, 1170, 1103 y $950 \mathrm{~cm}^{-1}$, para el octavo y décimo lavado estas bandas no están presentes con lo que se puede afirmar que el proceso de lavado con etilendiamina permite eliminar el ión sulfato del sistema. 


\subsubsection{Difracción de rayos $X$.}

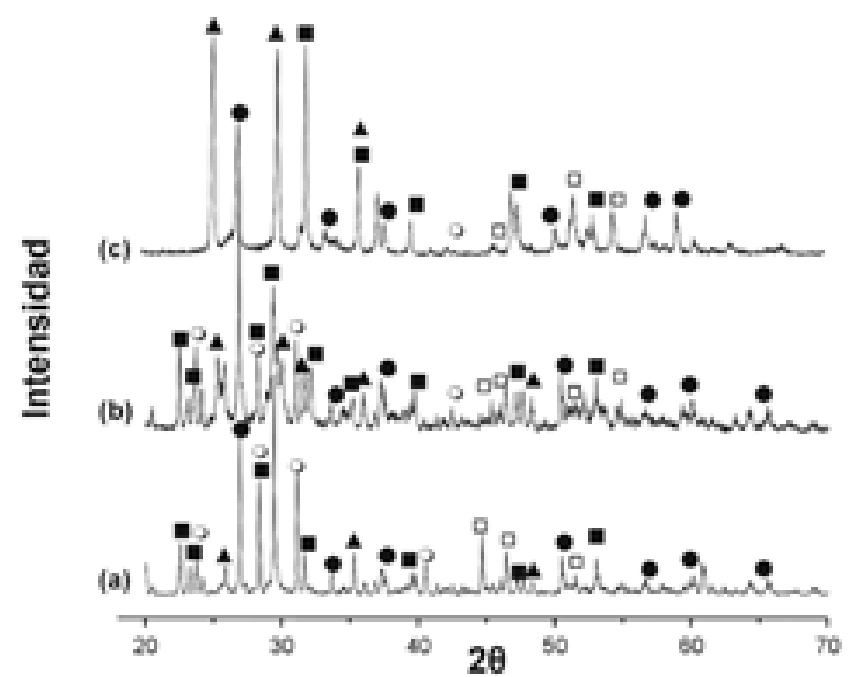

Fig. 4- Difractogramas de rayos $X$ correspondientes a muestras sólidas obtenidas a diferentes valores de $\mathrm{pH}$ (a) 3.97, (b) 6.40 y (c) 9.35 .

[- $\left.\mathrm{SnO}_{2} ; \square \mathrm{NH}_{4} \mathrm{NO}_{3} ; \mathrm{O}(\mathrm{NH} 4)_{2} \mathrm{SO}_{4} ; \mathrm{Sn}_{3} \mathrm{O}(\mathrm{OH})_{2} \mathrm{SO}_{4} ; \Delta \mathrm{SnO}\right]$.

En la figura 4 se muestran los difractogramas correspondientes a sólidos obtenidos del sistema con una concentración $0,3 \mathrm{M}$ de sulfato de estaño y $0,1 \mathrm{M}$ de $\mathrm{HNO}_{3}$ a diferentes valores de $\mathrm{pH}$, los cuales indican que las principales fases cristalinas presentes en ellos son las siguientes: $\mathrm{SnO}$ (PDF77-2296), $\mathrm{SnO}_{2}$ (PDF41-1445), $\mathrm{Sn}_{3} \mathrm{O}(\mathrm{OH})_{2} \mathrm{SO}_{4}$ (PDF301376), $\mathrm{NH}_{4} \mathrm{NO}_{3}$ (PDF73-1518), y $\left(\mathrm{NH}_{4}\right)_{2} \mathrm{SO}_{4}$ (PDF84-0127). Como se puede observar en la figura 4, para un $\mathrm{pH}$ de 6,40 la casiterita $\mathrm{SnO}_{2}$ es una de las principales fases cristalinas y el $\mathrm{Sn}_{3} \mathrm{O}(\mathrm{OH})_{2} \mathrm{SO}_{4}$ ya no está presente en gran cantidad como para los valores de $\mathrm{pH} 3,97$ y 9,35.

Con el fin de observar el efecto que tiene el proceso de lavado del precipitado con una solución $0.005 \mathrm{M}$ de etilendiamina, sobre la transformación de fases de los compuestos que existen en el sistema de estaño, se utilizó DRX, (figura 5).

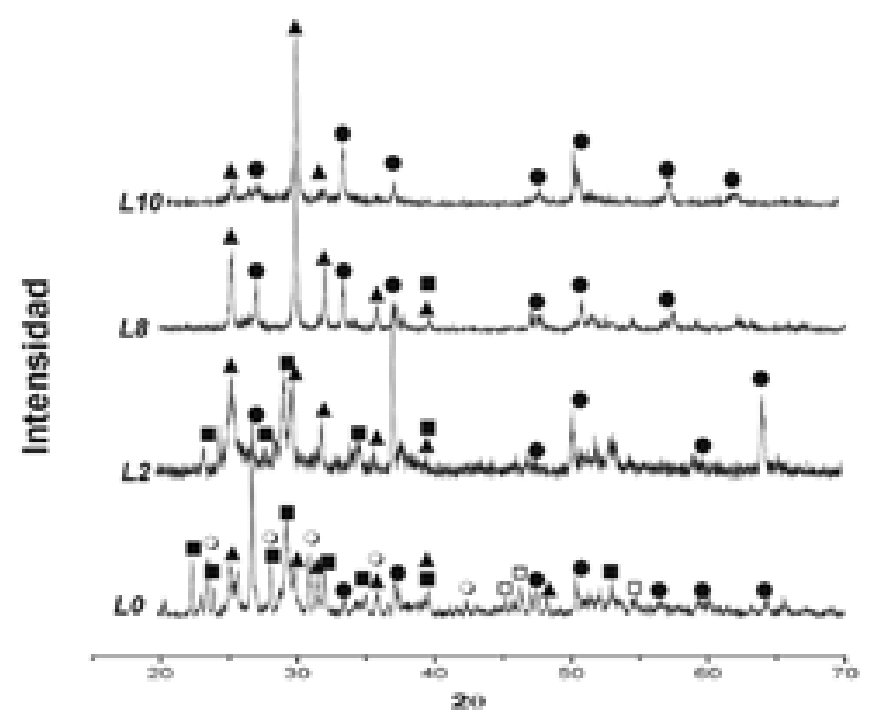

Fig. 5- Difractogramas de rayos $\mathrm{X}$ correspondientes a muestras sólidas obtenidas a $\mathrm{pH}$ 6.40, sometidas a diferentes procesos de lavados.

$\left[\bullet \mathrm{SnO}_{2} ; \square \mathrm{NH}_{4} \mathrm{NO}_{3} ; \mathrm{O}(\mathrm{NH} 4)_{2} \mathrm{SO}_{4} ; \mathrm{Sn}_{3} \mathrm{O}(\mathrm{OH})_{2} \mathrm{SO}_{4} ; \mathbf{\Delta} \mathrm{SnO}\right]$.
Como se puede observar en la figura 5, en la muestra sólida del segundo lavado está presente la fase $\mathrm{Sn}_{3} \mathrm{O}(\mathrm{OH})_{2} \mathrm{SO}_{4^{\prime}}$ pero para el octavo y décimo lavado, la etilendiamina elimina la presencia de las fases $\mathrm{Sn}_{3} \mathrm{O}(\mathrm{OH})_{2} \mathrm{SO}_{4}$ y $\left(\mathrm{NH}_{4}\right)_{2} \mathrm{SO}_{4^{\prime}}$, resultados que reiteran lo observado en los espectros FTIR. Además, las únicas fases cristalinas presentes son la casiterita, $\mathrm{SnO}_{2}$ y la romarchita, $\mathrm{SnO}$, siendo esta última la fase cristalina más importante. Tanto los resultados de FTIR como de DRX verifican la posibilidad de obtener óxido de estaño, $\mathrm{SnO}$ o $\mathrm{SnO}_{2}$, a temperatura ambiente, solamente lavando el precipitado obtenido a $\mathrm{pH} 6,4$ con una solución $0.005 \mathrm{M}$ de etilendiamina.

Tal comolo indican los resultados de FTIR y DRX, la muestra sólida correspondiente al décimo lavado con etilendiamina no presenta sulfato en su estructura. Este resultado se puede justificar considerando la formación de sales de amonio del tipo $\left(\mathrm{CH}_{3} \mathrm{CH}_{2}\right)_{2} \mathrm{NH}_{2}^{+} \mathrm{HSO}_{4}^{-}$durante el proceso de lavado (34) proceso que se esta estudiando actualmente en el laboratorio del grupo CYTEMAC.

\subsubsection{Microscopía electrónica de transmisión. (MET)}

La figura 6 muestra fotografías obtenidas con MET del polvo cerámico sintetizado del sistema $\mathrm{SnSO}_{4}-\mathrm{NH}_{3}-\mathrm{H}_{2} \mathrm{O}$, con una concentración $0.3 \mathrm{M}$ de sulfato de estaño a un valor de $\mathrm{pH} 6.40$.

En la figura 6(a) se observa la fotografía de la muestra sin tratamiento químico, es decir sin proceso de lavado, e ilustra que las partículas son de pequeño tamaño, $20 \mathrm{~nm}$, es evidente la formación de aglomerados de partículas debido a la acción de fuerzas superficiales, es mas claro si se observa la figura 6(b), que es la muestra al octavo lavado y el tamaño de la barra de la fotografía es de 500nm, por otro lado, también se puede ver regiones donde las partículas están mas sueltas, en la figura 6(c) es más evidente este efecto, en está foto se muestra la microscopía de la muestra al décimo lavado, donde ya no está presente el ion sulfato y se observa menos cantidad de aglomerados.

\section{CONCLUSIONES}

De este trabajo se puede concluir lo siguiente:

1.- El método de precipitación controlada permite obtener polvos cerámicos de óxido de estaño, $\mathrm{SnO}$ y $\mathrm{SnO}_{2}$ con tamaño nanométrico. El conocimiento de los diferentes fenómenos fisicoquímicos que ocurren durante la formación de las partículas, adquirido al analizar las curvas de valoración potenciométrica y caracterizando la fase sólida del sistema, permite obtener polvos cerámicos un tamaño aproximado de partícula de $20 \mathrm{~nm}$, además permite garantizar la reproducibilidad de este método de síntesis.

2.- Las principales fases cristalinas presentes en los sólidos obtenidos del sistema sulfato de estaño con una concentración

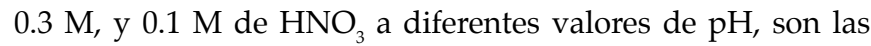
siguientes: $\mathrm{SnO}, \mathrm{SnO}_{2}, \mathrm{Sn}_{3} \mathrm{O}(\mathrm{OH})_{2} \mathrm{SO}_{4}, \mathrm{NH}_{4} \mathrm{NO}_{3}$ y $\left(\mathrm{NH}_{4}\right)_{2} \mathrm{SO}_{4}$. Después de lavar las muestras con agua se elimina la presencia del ión sulfato tal como se observó con FTIR; las bandas a 1170,1103 y $950 \mathrm{~cm}^{-1}$ correspondientes a los complejos de sulfato, ya no están presentes en el espectro. Estos resultados son verificados con DRX tal que en los difractogramas correspondientes a estas muestras no están presentes las fases cristalinas $\mathrm{Sn}_{3} \mathrm{O}(\mathrm{OH})_{2} \mathrm{SO}_{4}$ y $\left.\left(\mathrm{NH}_{4}\right)_{2} \mathrm{SO}_{4}\right)$ que afectan las 

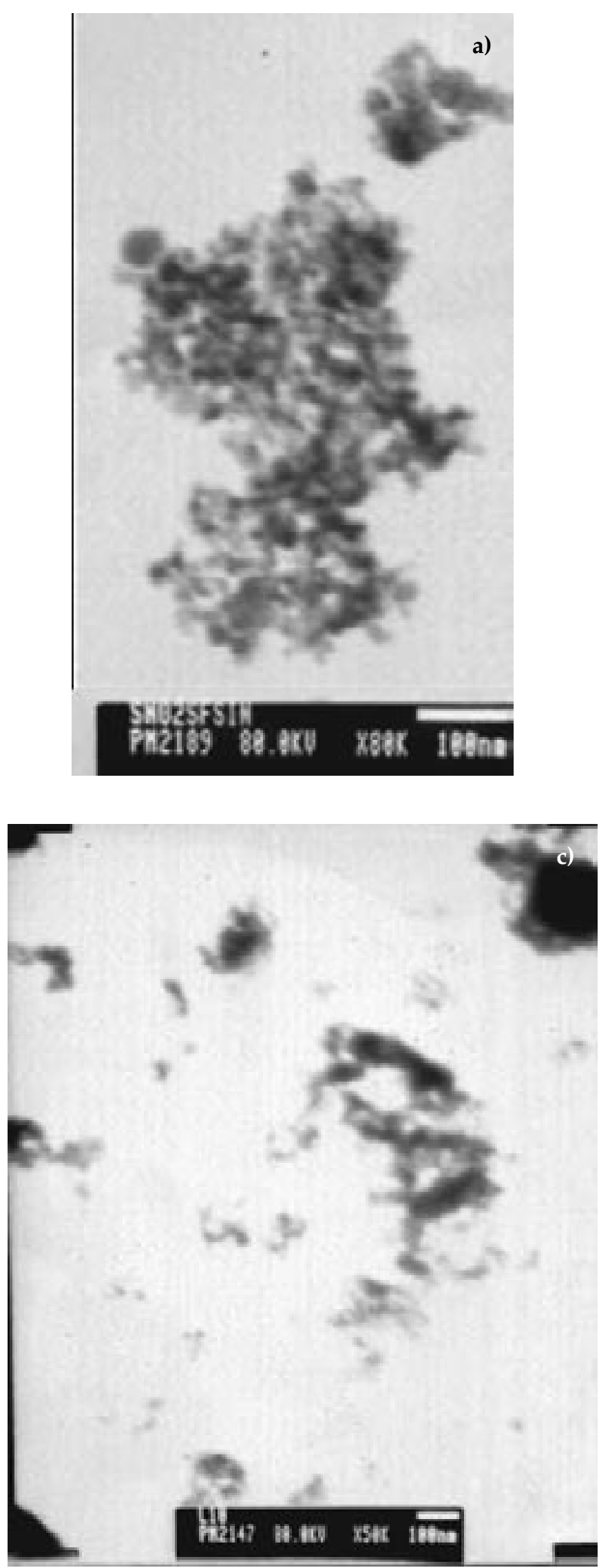

Fig. 6- Fotografías obtenidas con MET de los polvos cerámicos del sistema $0.3 \mathrm{M}$ de $\mathrm{SnSO} 4$, sintetizados a un valor de $\mathrm{pH} 6.40$ y tratados térmicamente a $60^{\circ} \mathrm{C}$; (a) sin lavados; barra $=100 \mathrm{~nm}$, (b) al octavo lavado; barra $=500 \mathrm{~nm}$ y (c) décimo lavado; barra $=100 \mathrm{~nm}$.

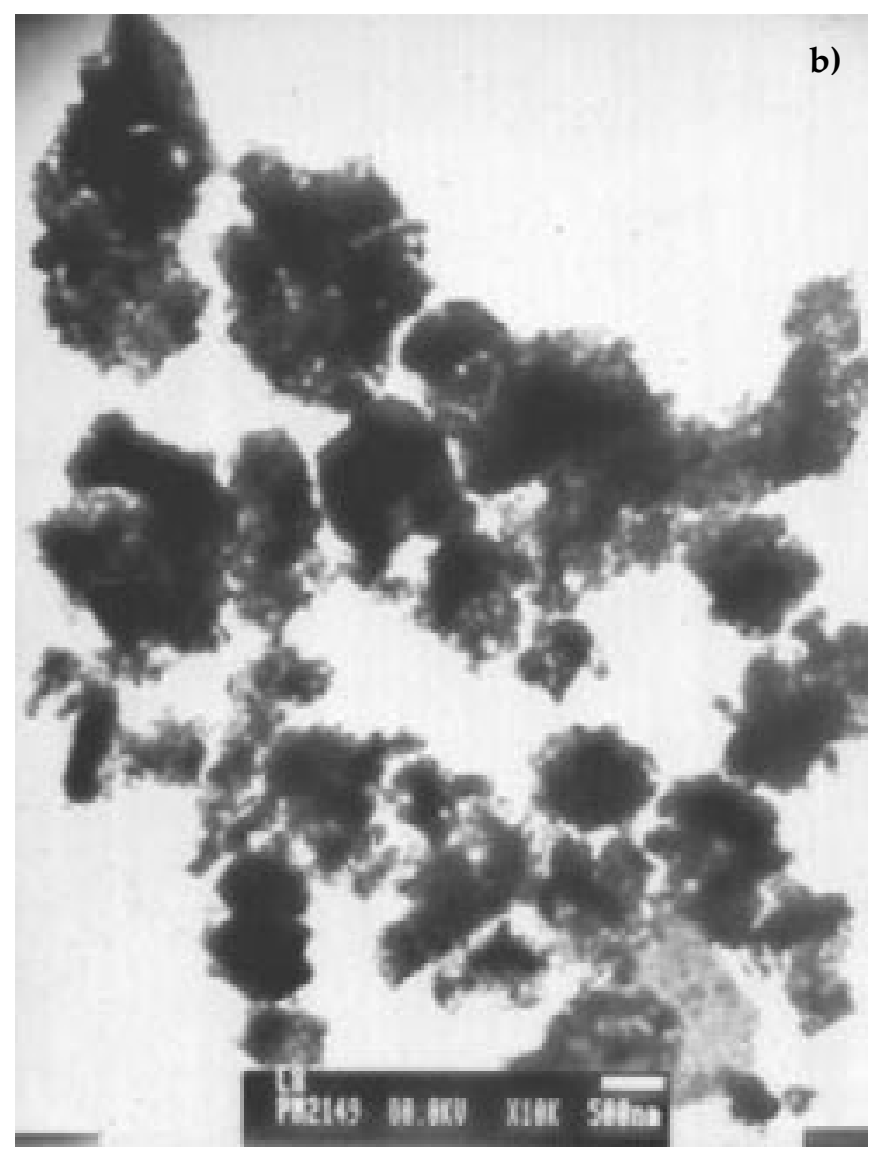

características finales del material en polvo y por lo tanto de sus aplicaciones.

3.- Por medio de microscopía electrónica de transmisión, se observa que los polvos cerámicas al ser tratados con una solución 0,005M de etilendiamina, se encuentran mas sueltos que los que no tienen lavados, esto se puede deber a que en estas muestras se ha eliminado el ion sulfato.

Con base en lo mencionado se puede concluir, de manera general, que es posible obtener óxido de estaño nanométrico, una mezcla de $\mathrm{SnO}_{2}$ y $\mathrm{SnO}$ donde está última fase cristalina es la más abundante, a temperatura ambiente de una solución de $\mathrm{SnSO}_{4}$, solamente lavando el precipitado obtenido a $\mathrm{pH}$ 6,4 con una solución $0.0005 \mathrm{M}$ de etilendiamina.

\section{AGRADECIMIENTOS}

Este proyecto fue realizado en el marco del proyecto COLCIENCIAS código 1103-14-17900. Los autores agradecen al proyecto PROALERTA VIII.13 de la Red CyTED de Materiales Electrocerámicos, por la ayuda económica brindada a la Srta. Alejandra Montenegro para que pudiera realizar su pasantía en la División Cerámicos del Instituto de Investigaciones en Ciencia y Tecnología de Materiales-INTEMA / Mar del PlataArgentina

\section{BIBLIOGRAFÍA}

1. K. J. Klanbunde. «Nanoscale Materials in chemistry», John Wiley \& Sons, Inc., New York, 2001.

2. A. S. Edelstein, R. C. Cammarata. «Nanomaterials: Synthesis, Propierties and aplications». Institute of Physics publishing Ltd, Series in Micro and Nanoscience and technology, Bristol, 2002. 
3. E. Matijevic. "preparation and properties of uniform size colloids». Chem. Mater., 5, 412, (1993).

4. E. Matijevic. «Monodidpersed colloids: Art and science». Langmuir, 2, 12, (1986).

5. J. M. Jarzebski, J. P Marton. «Physical properties of $\mathrm{SnO}_{2}$ materials-II. Marton». Electrochem. Soc; 129, 299-310, (1976).

6. F. Huamán, L. Reyes, W. Estrada, A Gutarra. «Detección de vapor de etanol por modificación de la conductividad eléctrica del dióxido de estaño». Tecnia; 8, 77-83, (1998).

7. R. Delgado. «Sensores de gases basados en óxidos de estaño: una aproximación electroquímica». Tesis doctoral Universidad de Barcelona (2001).

8. V. Marques. «Influência do óxido de cromo nas propriedades varistoras do óxido de estanho». Tesis de Maestría. Instituto de química de Araraquara. UNESP (2003).

9. C. Ararat, J.A. Varela, J. E. Rodríguez. «Uso de métodos químicos para la obtención de polvos cerámicos del sistema $(\mathrm{Sn}, \mathrm{Ti}) \mathrm{O}_{2} »$. Bol. Soc. Esp. Ceram. V., 44, 4, 215-222, (2005).

10. K. L. Chopra, S. Major, P. K. Pandya. «Transparent conductors-A status review». Thin Solids Films; 102, 63-66, (1983).

11. K. Chatterjee, S. Chatterjee, A. Banerjee, M. Raut, N. Pal, H.S. Maiti. «The effect of palladium incorporation on methane sensitivity of antimony doped Tin». Mater. Chem. Phys; 81, 33-38, (2003).

12. J. G. Fagan; V. R. Amarakon. «Realiability and reproducibility of ceramic sensors - III». AM. Ceram. Soc. Bull; 72, 119-129, (1993).

13. J. Zhang, L. Gao, J. «Synthesis and characterization of nanocrystalline tin oxide by sol-gel method». Solid Stat. Chem. 177 1425-1430 (2004).

14. C. J. Brinker, G. W. Scherer. «Sol-gel Science: The physics and chemistry of sol-gel processing». Academic Press, San Diego, C.A. 1990.

15. J. P. Jolivet. «Metal Oxide Chemistry and synthesis: from solution to solid State». Wiley- VCH, Weinheim, 2000.

16. S. de Monredon, A. Cellot, F. Ribot, C. Sanchez, L. Armelao, L. Gueneaw, L. Delattre. "Synthesis and characterization of crystalline tin oxide nanoparticles». J. Mater. Chem., 12 2396-2400 (2002).

17. K. Ch. Song, Y. Kang. «Preparation of high surface area tin oxide powders by a homogeneus precipitation method». Materials letters, 42 283-289 (2000).

18. N. Sergent, P. Gélin, L. Périer- Camby, h. Praliaud, G. Thomas. «Preparation and characterization of high surface area stannic oxides: structural textural and semiconducting propierties». Sensors and actuactors B, 84 176-188 (2002).
19. M. Ristic, M. Ivanda, S. Popovic, S. Music. «Dependence of nanocrystalline $\mathrm{SnO}_{2}$ particle size on synthesis route». J. Non- Cryst. Solids 303 270-280 (2002).

20. P. Smith. «Quemistry of tin», Blackie academy professional 2 ed.

21. M. Batzill, U. Diebold. «The surface and materials science of tin oxide». Progress in surface science, 79 (2-4) 47-154 (2005).

22. L. A Errico «Ab initio FP-LAPW study of the semiconductors $\mathrm{SnO}$ and $\mathrm{SnO}_{2}$ ». Physica B: Condensed Matter, 389 (1) 140-144 (2007).

23. A. Hayashi, M. Nakai, M. Tatsumisago, T. Minami, Y. Himei, Y. Miura, M. Katada. «Structural investigation of $\mathrm{SnO}-\mathrm{B}_{2} \mathrm{O}_{3}$ glasses by solid-state NMR and X-ray photoelectron spectroscopy». J. of Non-Crystalline Solids, 306 (3), 227-237 (2002)

24. J. Chouvin, J. Olivier-Fourcade, J. C. Jumas, B. Simon, Ph. Biensan, F. J. Fernández Madrigal, J. L. Tirado, C. Pérez Vicente. «SnO reduction in lithium cells: study by X-ray absorption, 119Sn Mössbauer spectroscopy and X-ray diffraction». J. of Electroanalytical Chem.. 494 (2) 136-146 (2000)

25. A. Montenegro. «Obtención de sensores de gas a partir de los sistemas SnBi y Sn-Sb» Trabajo de grado, Ing. Física. Universidad del Cauca, 2006.

26. N. N. Greenwood, A. Earnshaw, "Chemistry of the elements». ButterworthHeinemann Ltd. (1996).

27. C.F. Baes, R. E. Mesiner, «The hydrolysis of cations». Wiley-Interscience, John Wiley \& sons, Inc. New York, 1976.

28. C. K. Song, Y. Kang. «Preparation of high surface area tin oxide powders by a homogeneus precipitation method». Materials letters 42 283-289 (2000).

29. V. M. Jiménez, A. Fernandez, J. P. Espinos. «Structural characterization of partially amorphous $\mathrm{SnO}_{2}$ nanoparticles by factor análisis of XAS and FTIR spectra». Solid State Ionics 116 117-127 (1999).

30. Y. C. Farmer. «The infrared spectra of minerals». Minerologycal Society. (1974).

31. K. Nakamoto. «Infrared and Raman spectra of inorganic and coordination compounds». John Wiley \& sons Ltd, (1986).

32. M. Ocaña, E. Matijevic. «Well-defined colloidal tin (IV) oxide particles». J. Mater. Res. 5(5) 1083-1091 (1990).

33. N. Sergnt, P. Celine. «Preparation and characterization of high surface area stannics oxides». Sensors and Actuactors A 84, 176-188 (2002).

34. T.W.G. Solomons. "Organic chemistry» Fifth edition, Jhon Wiley \& Sons, $\mathrm{InC}_{4^{\prime}} 1992$.

Recibido: 24.11 .06

Aceptado: 23.03 .07 